\title{
Brueckner-Hartree-Fock study of circular quantum dots
}

\author{
A. Emperador \\ Departament de Física i Enginyeria Nuclear, Campus Nord B4-B5, Universitat Politècnica de Catalunya, E-08034 Barcelona, Spain \\ E. Lipparini* \\ Departament ECM, Facultat de Física, Universitat de Barcelona, Diagonal 647, E-08028 Barcelona, Spain
}

\author{
Ll. Serra \\ Departament de Física, Universitat de les Illes Balears and Institut Mediterrani d'Estudis Avançats IMEDEA (CSIC-UIB), \\ E-07122 Palma de Mallorca, Spain
}

(Received 30 November 2005; revised manuscript received 14 February 2006; published 22 June 2006)

\begin{abstract}
We calculate ground state energies in the Brueckner-Hartree-Fock theory for $N$ electrons (with $N \leqslant 20$ ) confined to a circular quantum dot and in presence of a static magnetic field. Comparison with the predictions of Hartree-Fock, local-spin-density and exact configuration-interaction theories is made. We find that the correlations taken into account in Brueckner-Hartree-Fock calculations give an important contribution to the ground state energies, especially in strongly confined dots. In this high-density range, corresponding in practice to self-assembled quantum dots, the results of Brueckner-Hartree-Fock calculations are close to the exact values and better than those obtained in the local-spin-density approximation.
\end{abstract}

DOI: 10.1103/PhysRevB.73.235341

PACS number(s): 73.21.La, 71.10.-w

\section{INTRODUCTION}

The Hartree and Hartree-Fock (HF) mean field approaches have been extensively used in the past to study atomiclike properties of semiconductor quantum dots such as, e.g., those measured in conductance and capacitance experiments. ${ }^{1-4}$ Symmetry restricted approximations, as well as spin and/or space unrestricted HF solutions have been analyzed. ${ }^{5-10}$ These methods give results that are satisfactory for a qualitative understanding of many properties of these systems. However, comparison with exact configurationinteraction diagonalization ${ }^{11-17}(\mathrm{CI})$ and quantum Monte Carlo $^{18-22}$ (QMC) studies show discrepancies in the total energies that are substantial on the relevant energy scale.

The discrepancies become even larger in the presence of a constant magnetic field which magnifies the importance of correlations. However, at high magnetic field QMC calculations are imprecise due to the fixed phase approximation and the exact diagonalization techniques require a number of configurations which increases exponentially with the number of particles, making the calculation unpractical for more than ten particles. An alternative method that has been shown to be quite accurate for cases in which HF yields broken symmetry solutions is that of symmetry restoration by projection, ${ }^{23}$ although its complexity also increases very rapidly with the number of particles. The ground state properties of many electrons quantum dots can also be calculated with local-spin-density approximation (LSDA) and current density functional methods. ${ }^{24-26}$ These approaches are based on energy functionals obtained through fits and interpolations of QMC results for the spin polarized and unpolarized twodimensional electron gases that do not include a magnetic field and, moreover, provide a single particle spectrum whose physical interpretation is not clear. Nevertheless, LSDA has given good results for quantum dots with low electronic densities, having Wigner-Seitz radius $r_{s} \gtrsim 1$. These are the typical densities of quantum dots made by etching ${ }^{3}$ or by lithography, ${ }^{1}$ usually over a GaAs substrate. Another class of quantum dots are the self-assembled ones, ${ }^{27,28}$ with high electronic densities $\left(r_{s}<0.5\right)$ and great practical interest due to technological applications such as, e.g., in semiconductor lasers. $^{29}$ They are usually formed over InAs substrates, have small diameters and contain strongly confined electronic states. For systems so distant from the homogeneous conditions one should not expect the LSDA to give accurate predictions and, indeed, most calculations on InAs dots have been made using non-DFT-based schemes. ${ }^{30-32}$

In this paper we employ the Brueckner-Hartree-Fock (BHF) method ${ }^{33,34}$ to describe electrons in quantum dots because it yields good ground state energies at a computational cost that grows in a relatively modest way with the number of particles, as compared to CI and QMC (in its most accurate versions such as diffusion QMC). This method has been extensively applied in the past to study nuclei ${ }^{35}$ and metallic clusters. ${ }^{36}$ Also, a related scheme based on Bethe-Goldstone equations has been used to study small excitonic complexes in quantum dots. ${ }^{37}$ The underlying assumption of the BHF theory is a description in terms of independent pairs of particles. For each pair, interaction affects the way in which the two particles scatter, while the rest of particles are considered noninteracting and their influence is only through the Pauli exclusion principle. It is well known that the exclusion principle imposed by the Fermi sea induces a modification of the pair wave function at short interparticle distances, inducing the so-called "wound" in the wave function. Therefore, the BHF theory describes short range statistical correlations and will miss, by construction, long range correlations associated with collective motions. ${ }^{33,34}$

Here we compare BHF energies for electrons in quantum dots with the available results from methods attempting a direct solution of the many body Schrödinger equation like CI diagonalization and QMC, as well as with approximate models such as LSDA and HF. We find that BHF is always more accurate than HF. It recovers the exact result in the 
strong-confinement limit and at high magnetic fields, when the maximum-density-droplet (MDD) is formed, it yields more accurate energies than the LSDA ones. Furthermore, in the case of electronic densities typical of self-assembled quantum dots, BHF also gives much better results than LSDA even in the absence of magnetic fields.

\section{THE BHF SCHEME}

This section recalls some essential points of the BHF theory deriving several relevant equations and quoting others without proof. The restriction to circular symmetry solutions and its practical implications are also discussed.

\section{A. Theory}

Let us consider a system of $N$ electrons with Hamiltonian

$$
H=\sum_{i}^{N} h_{0}(i)+\sum_{i<j}^{N} v_{i j}
$$

where $h_{0}=t_{i}+v_{\text {ext }}\left(r_{i}\right), t_{i}$ is the single particle kinetic energy which can include the effect of the magnetic field, $v_{\text {ext }}$ the confining potential and $v_{i j}=e^{2} / \epsilon\left|\mathbf{r}_{i}-\mathbf{r}_{j}\right|$, with $\boldsymbol{\epsilon}$ the dielectric constant. The ground state $|\Psi\rangle$ is solution of the many-body Schrödinger equation

$$
(H-E)|\Psi\rangle=0,
$$

where $E$ is the ground state energy. Let us now consider an independent particle model (the HF model in the following) where the eigenstates $\left|\Phi_{n}\right\rangle$ are Slater determinants solutions of the equation

$$
\left(H_{H F}-W_{n}\right)\left|\Phi_{n}\right\rangle=0,
$$

where $H_{H F}=\mathcal{C}+\Sigma_{i}^{N}\left(h_{0}(i)+U_{i}\right)$, with $U_{i}$ the HF potential and $\mathcal{C}$ a constant. The HF ground state determinant and energy are given by $\left|\Phi_{0}\right\rangle \equiv|H F\rangle$ and $W_{0} \equiv E_{H F}$, respectively.

The residual interaction $V_{\text {res }}$ fulfills

$$
\begin{gathered}
H=H_{H F}+V_{\text {res }}, \\
V_{\text {res }}=\sum_{i<j}^{N} v_{i j}-\sum_{i}^{N} U(i)-\mathcal{C} .
\end{gathered}
$$

HF theory yields the following general matrix elements:

$$
\begin{gathered}
\left\langle H F\left|V_{\text {res }}\right| H F\right\rangle=0, \\
\left\langle H F\left|V_{\text {res }}\right| m i^{-1}\right\rangle=0, \\
\left\langle H F\left|V_{\text {res }}\right| m n i^{-1} j^{-1}\right\rangle \neq 0,
\end{gathered}
$$

where we have used the standard notation for particle-hole (ph) excitations, i.e., indexes $i, j(m, n)$ refer to orbitals below (above) the Fermi energy and $\left|m i^{-1}\right\rangle$ is the Slater determinant obtained promoting one electron from orbital $i$ to orbital $m$ in $|H F\rangle$. Note that only $2 p-2 h$ excitations yield nonvanishing transition matrix elements since the two-body nature of $V_{\text {res }}$ ensures that matrix elements between determi- nants differing in more than two orbitals will again vanish. Another immediate consequence from Eqs. (6)-(8) is that $E_{H F}=\langle H F|H| H F\rangle$.

We can write

$$
|\Psi\rangle=|H F\rangle+\sum_{n \neq 0} a_{n}\left|\Phi_{n}\right\rangle
$$

From Eqs. (2), (4), and (9) one easily finds that

$$
\left(H_{H F}-E\right)\left(|H F\rangle+\sum_{n \neq 0} a_{n}\left|\Phi_{n}\right\rangle\right)+V_{r e s}|\Psi\rangle=0
$$

Multiplying Eq. (10) by $\langle H F|$ on the left one gets

$$
E=E_{H F}+\left\langle H F\left|V_{r e s}\right| \Psi\right\rangle \text {. }
$$

If multiplying by $\left\langle\Phi_{n}\right|$ one finds $a_{n}=\frac{\left\langle\Phi_{n}\left|V_{\text {res }}\right| \Psi\right\rangle}{E-W_{n}}$ and hence the following implicit equation is obtained:

$$
|\Psi\rangle=|H F\rangle+\sum_{n \neq 0} \frac{\left\langle\Phi_{n}\left|V_{r e s}\right| \Psi\right\rangle}{E-W_{n}}\left|\Phi_{n}\right\rangle .
$$

This equation can be solved by iteration taking as starting energy the HF one

$$
|\Psi\rangle=|H F\rangle+\sum_{n \neq 0} \frac{\left\langle\Phi_{n}\left|V_{r e s}\right| H F\right\rangle}{E_{H F}-W_{n}}\left|\Phi_{n}\right\rangle+\cdots
$$

yielding for the energy

$$
E=E_{H F}+\sum_{n \neq 0} \frac{\left|\left\langle\Phi_{n}\left|V_{r e s}\right| H F\right\rangle\right|^{2}}{E_{H F}-W_{n}}+\cdots
$$

At the first order in $V_{\text {res }}$ this equation gives a result for the energy which coincides with the one of first order perturbation theory, summing all the orders we get the correlation energy in the ladder approximation. This is more clear defining the $G$-matrix by the relation

$$
G|H F\rangle=V_{\text {res }}|\Psi\rangle
$$

We then get the Bethe-Goldstone implicit equation for $G$

$$
G=V_{\text {res }}+\sum_{n \neq 0} V_{r e s} \frac{\left|\Phi_{n}\right\rangle\left\langle\Phi_{n}\right|}{E-W_{n}} G
$$

and from Eq. (11)

$$
E=E_{H F}+\langle H F|G| H F\rangle=E_{H F}+\sum_{n \neq 0} \frac{\left\langle H F\left|V_{r e s}\right| \Phi_{n}\right\rangle\left\langle\Phi_{n}|G| H F\right\rangle}{E-W_{n}}
$$

Only $2 p-2 h$ determinants yield a nonvanishing contribution to the sum of Eq. (17), which can thus be reduced to a sum of two-body matrix elements. Assuming $E=E_{H F}$ on the righthand side, as in the ladder approximation, one has 
TABLE I. Ground state energies for the dots with $2 \leqslant N \leqslant 13$ computed by HF, BHF, LSDA, QMC, and CI methods. The energies $E$ in units of the confinement energy $\hbar \omega_{0}$ are tabulated as $E^{\prime}=E / \hbar \omega_{0}$. A fixed value $R=1.89$ of the interaction-toconfinement ratio has been used.

\begin{tabular}{lccccc}
\hline \hline$N$ & $E_{\mathrm{HF}}^{\prime}$ & $E_{\mathrm{BHF}}^{\prime}$ & $E_{\mathrm{LSDA}}^{\prime}$ & $E_{\mathrm{QMC}}^{\prime}$ & $E_{\mathrm{CI}}^{\prime}$ \\
\hline 2 & 4.078 & 3.832 & 3.739 & 3.650 & 3.646 \\
3 & 8.589 & 8.289 & 8.082 & 7.979 & 7.957 \\
4 & 13.94 & 13.63 & 13.16 & 13.26 & 13.06 \\
5 & 20.96 & 20.38 & 19.91 & 19.76 & 19.53 \\
6 & 28.70 & 27.72 & 27.27 & 27.14 & 26.82 \\
7 & 37.46 & 36.61 & 35.96 & 35.86 & \\
8 & 46.93 & 46.11 & 45.46 & 45.32 & \\
9 & 57.89 & 56.71 & 55.79 & 55.64 & \\
10 & 69.29 & 67.75 & 67.00 & 66.86 & \\
11 & 81.54 & 79.86 & 78.96 & 78.86 & \\
12 & 94.82 & 94.36 & 91.71 & 91.64 & \\
13 & 108.64 & 106.36 & 105.50 & 105.32 & \\
\hline \hline
\end{tabular}

$$
E=E_{H F}+\frac{1}{2} \sum_{i j m n} \frac{\langle i j|v| m n\rangle}{\epsilon_{i}+\epsilon_{j}-\epsilon_{m}-\epsilon_{n}} \times(\langle m n|g| i j\rangle-\langle m n|g| j i\rangle),
$$

where the $\epsilon_{\alpha}$ are the HF single particle energies and we have associated the $G$ matrix with an effective two-body interaction $g$.

In order to have a practical computational scheme it remains now to specify the two-body matrix elements of $g$ in Eq. (18). This is accomplished within the BHF independentpair model, ${ }^{33}$ where the off-diagonal matrix elements are found from

$$
\langle m n|g| i j\rangle=\langle m n|v| i j\rangle+\sum_{p q} \frac{\langle m n|v| p q\rangle\langle p q|g| i j\rangle}{\epsilon_{i}+\epsilon_{j}-\epsilon_{p}-\epsilon_{q}} .
$$

The ground state energy of Eq. (18) with the matrix elements obtained from Eq. (19) is the BHF energy which sums all the ladder diagrams corresponding to the iterated solutions of Eq. (14).

\section{B. Circular symmetry restriction}

In this work we restrict to circular symmetry cases, where the HF orbitals can be factorized as

$$
\langle\mathbf{r} \sigma \mid i\rangle \equiv R_{n_{i} m_{i}}(r) \frac{e^{i m_{i} \theta}}{\sqrt{2 \pi}} \chi_{\mu_{i}}(\sigma),
$$

where $n_{i}=0,1, \ldots, m_{i}=0, \pm 1, \ldots$, and $\mu_{i}= \pm 1 / 2$ are the principal, angular momentum, and spin quantum numbers, respectively. In this situation the angular and spin parts of the two-body matrix elements yield selection rules on the corresponding quantum numbers and the matrix elements reduce to

$$
\begin{aligned}
\langle a b|v| c d\rangle= & \delta_{m_{a}+m_{b}, m_{c}+m_{d}} \delta_{\mu_{a} \mu_{c}} \delta_{\mu_{b} \mu_{d}} \\
& \times I_{r}\left(R_{n_{a} m_{a}}, R_{n_{b} m_{b}}, R_{n_{c} m_{c}}, R_{n_{d} m_{d}}\right),
\end{aligned}
$$

where $I_{r}$ is a radial integral that we compute numerically. Note that through Eq. (19) the same angular momentum selection rules apply to $\langle a b|g| c d\rangle$ and that both matrix elements are real.

The two-body matrix elements of $g$ required for the evaluation of the total energy, Eq. (18), are found by solving Eq. (19) as a linear system for the unknowns $\langle m n|g| i j\rangle$. For each pair $i j$ we have an independent linear system and the above mentioned selection rules are very important since they allow a big reduction in the number of effectively coupled equations. Since the space of particle states must be truncated, the convergence of the calculation with the number of empty HF states has to be controlled. Another check of the numerical accuracy must be done regarding the number of radial points used in the evaluation of the integrals $I_{r}$ of Eq. (21).

\section{RESULTS}

Table I compares the energies of $B=0$ ground states of $N$-electron dots in BHF with the results of HF, LSDA (using the Tanatar-Ceperley parametrization for the correlation energy $^{38}$ ) and diffusion QMC. ${ }^{22}$ The external confinement is taken of parabolic type $v_{\text {ext }}(r)=m \omega_{0}^{2} r^{2} / 2$, with $m$ the electron effective mass. We refer all energies to the confinement energy $\hbar \omega_{0}$ and characterize the interaction strength by the repulsion-to-confinement ratio $R$, defined as

$$
R \equiv \frac{e^{2} /\left(\epsilon \ell_{0}\right)}{\hbar \omega_{0}}
$$

with $\ell_{0}$ indicating the oscillator length $\left(\hbar \omega_{0}=\hbar^{2} / m \ell_{0}^{2}\right)$. The results in Table I correspond to $R=1.89$. Taking, for instance, typical GaAs values $\epsilon=12.4$ and $m^{*}=0.067 m_{e}$ the chosen $R$ value would correspond to a confinement energy of $\hbar \omega_{0}=3.32 \mathrm{meV}$, which reproduces the experimental value of Ref. 3. The number of electrons is varied from $N=2$ to 13 .

At $B=0$ the BHF energies obviously improve the HF ones, although they are still appreciably higher than the QMC and LSDA values. This is due to the fact that in BHF long-range collective correlations are missed. The importance of short range correlations is expected to increase as the system is more tightly confined, for a fixed strength of the Coulomb repulsion and, thus, a better performance of $\mathrm{BHF}$ is expected when increasing the confinement strength. Indeed, this is shown to be the case in Fig. 1, where for $N=2$ and 6 at $B=0$ we have varied the value of $R$. The lower panels of this figure display the correlation energies, defined as usual by subtracting the HF value $E_{H F}$ from the total energy $E$, i.e., $E_{c o r r}=E-E_{H F}$. Note that although the correlation energy is globally reduced when the ratio decreases BHF accounts for a larger part of it. Actually, for $N=2 \mathrm{BHF}$ accounts for $71 \%$ of the full (CI) correlation energy when $R=1$ and $87 \%$ when $R=0.5$. The corresponding figures for $N=6$ are $64 \%(R=1)$ and $75 \%(R=0.5)$. These numerical results are thus showing that in the limit of small $R$ BHF 


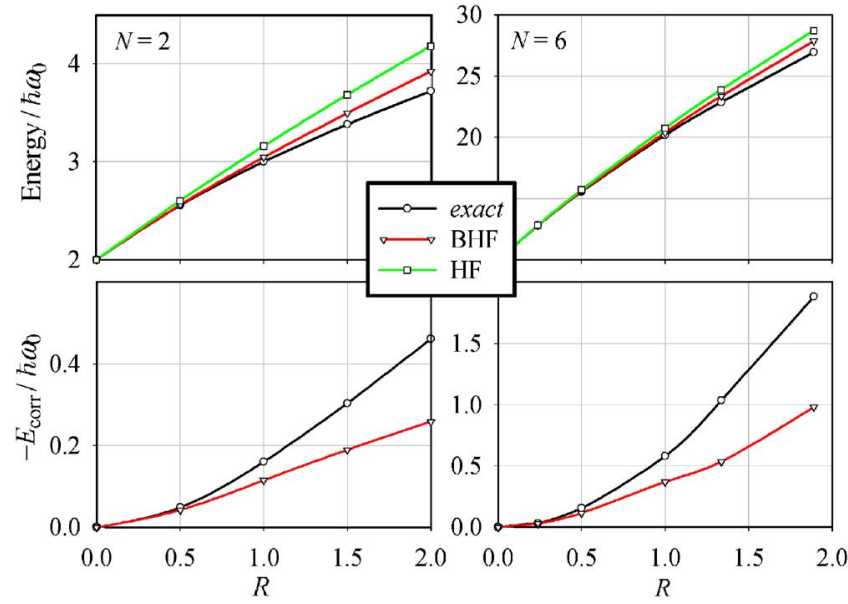

FIG. 1. (Color online) Upper panels: Total energies in different methods for two and six-electron dots as a function of the interaction-to-confinement ratio (see text). Lower: Correlation energies within each model for the same two dots.

converges to the exact correlation energy. It can be also seen from Fig. 1 that at a given value of $R$, BHF correlations for the $N=2$ dot are a somewhat larger piece of the total correlation energy than for $N=6$. We attribute this difference to a more important role of the collective effects leading to long range correlations not included in BHF for the six-electron dot.

We focus next on the influence of a magnetic field and how the BHF energies are affected by it. In the symmetric gauge, a magnetic field in the $z$ direction (perpendicular to the dot plane) induces a modification of the effective confinement from $\omega_{0}$ to $\Omega=\sqrt{\omega_{0}^{2}+\omega_{c}^{2} / 4}$, with $\omega_{c}$ the cyclotron frequency. For increasing magnetic fields one then expects short range correlations to be enhanced due to the stronger confinement and, therefore, an improved performance of the

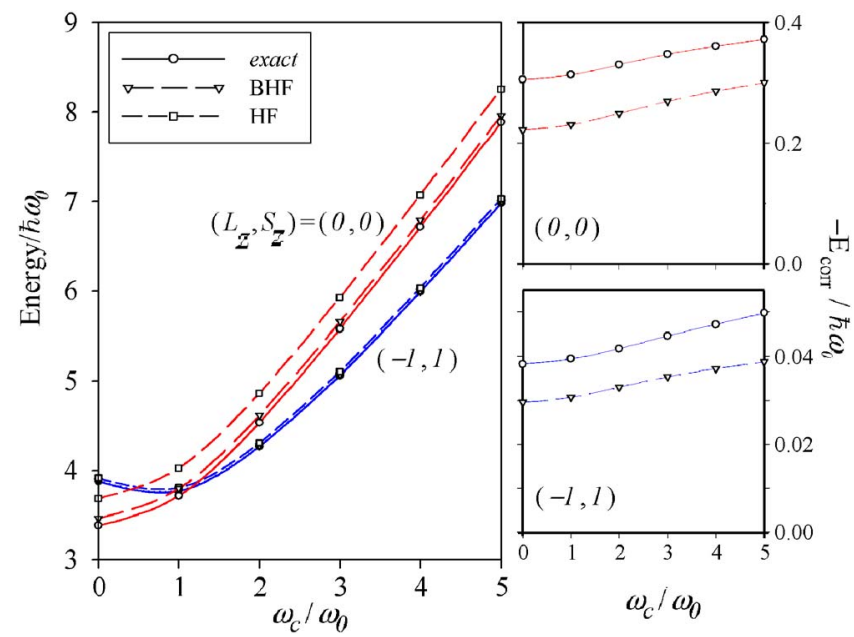

FIG. 2. (Color online) Left (right) panels show total (correlation) energies for the models and states indicated by the corresponding labels. The results correspond to the $N=2$ dot in a magnetic field, shown as a function of the cyclotron frequency (in units of $\left.\omega_{0}\right)$. The interaction-to-confinement ratio $R$ (see text) is chosen as $R=1.5$.

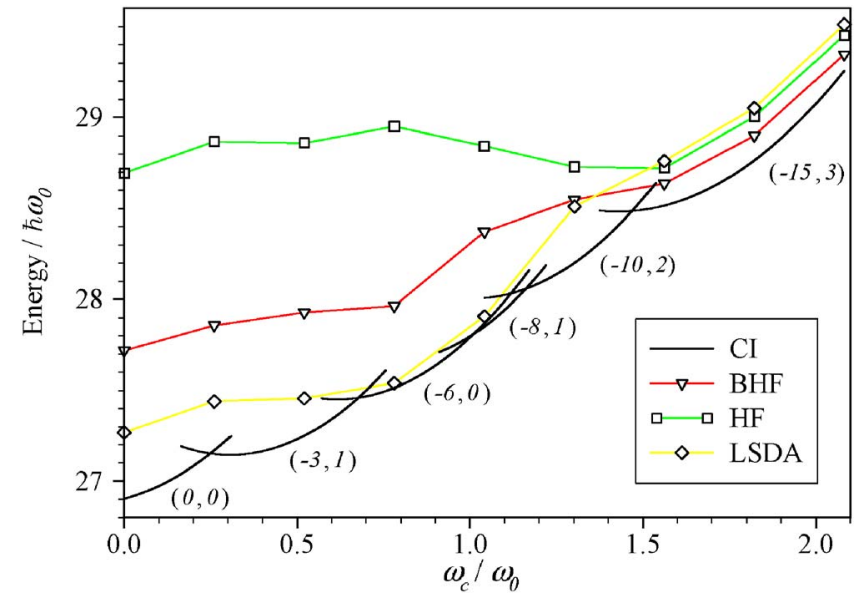

FIG. 3. (Color online) Evolution of the total energy of a sixelectron dot with the magnetic field. The different phases are indicated by the angular momentum labels $\left(L_{z}, S_{z}\right)$. A fixed value of the parameter $R=1.89$ has been used (see text).

BHF method. Figure 2 displays the evolution of the total energy with the ratio $\omega_{c} / \omega_{0}$ for a fixed value of $R=1.5$ and for a dot with 2 electrons. Note that the correlation energy is about an order of magnitude higher for the singlet than for the triplet. ${ }^{13}$ As expected, for increasing values of $\omega_{c} / \omega_{0}$ BHF is accounting for a higher part of the correlation energy, although the increase is rather moderate. For the singlet state BHF correlations range from $73 \%$ to $81 \%$ of the total correlation energy when $\omega_{c}$ goes from 0 to $5 \omega_{0}$. The evolution is even flatter for the triplet, where BHF correlations remain at $\approx 75 \%$ for all values of $\omega_{c}$. Note also that due to the sizable energy correction for the singlet, the singlet-triplet transition point is remarkably improved in BHF with respect to HF.

Figure 3 shows the evolution with magnetic field of the results for a six-electron dot with a fixed interaction-toconfinement ratio of $R=1.89$. As compared to the $N=2$ case, this dot shows a much richer phase diagram, with large variations in ground state angular momentum and spin when increasing the magnetic field. Most remarkable is the comparison of BHF and LSDA energies: While LSDA is clearly superior to BHF at low fields the situation is reversed when entering the fully polarized phase corresponding to the maximum-density-droplet (MDD). In the MDD region the LSDA energy is actually higher than the HF one and only BHF is able to provide an energy correction in the right direction with respect to HF; total BHF energies being approximately halfway of $\mathrm{CI}$ and $\mathrm{HF}$.

All the BHF results shown above have been obtained using a large enough space of empty HF states, always checking that for the given accuracy convergence in Eq. (18) has been achieved. In practice, we include the lowest $N_{p}$ particle states and repeat the calculation increasing this number. Figure 4 shows the evolution of the BHF energy with $N_{p}$, on scale proportional to $1 / N_{p}$, for three selected cases: The two upper panels correspond to six and nine electrons with the MDD configuration in strong magnetic field and a moderate confinement, while the lower panel shows the case of four electrons in very strong confinement and zero field. The results have been fitted with a polynomial including powers up 

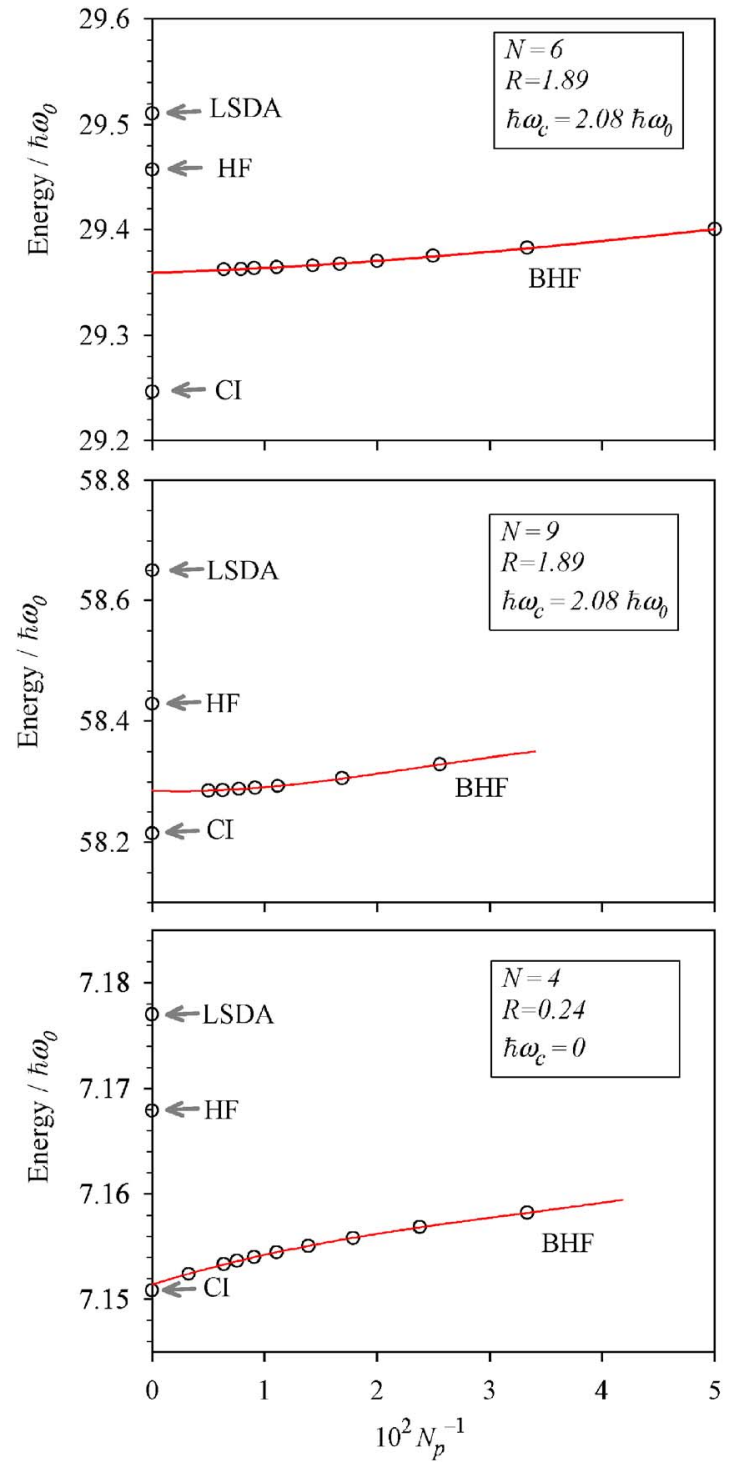

FIG. 4. (Color online) Evolution of the total BHF energy with the number $N_{p}$ of empty HF states included in the solution of Eq. (19). Each panel shows the results for a different quantum dot. The solid line is a cubic fit, in powers of $1 / N_{p}$, allowing extrapolation to the $N_{p} \rightarrow \infty$ limit. The LSDA, HF, and CI energies for each case are also shown for comparison.

to $1 / N_{p}^{3}$. In the chosen scale, the $N_{p} \rightarrow \infty$ limit is given by the intersection of the polynomial fit with the left vertical axis. It can be seen from Fig. 4 that the convergence is somewhat faster for the moderate confinement cases. However, in both examples the evolution with space dimension is quite smooth, indicating that the correlation energy builds up by gathering contributions from many states. The $N=9$ results of Fig. 4 provide additional support to our preceding conclusion from Fig. 3 that BHF performs much better than LSDA in the MDD region.

As already mentioned, for strong confinements the BHF energies are close to the exact values. Indeed, the $N=4$ results of Fig. 4 are very illustrative in this respect since the extrapolated BHF energy essentially coincides with the CI result. This strongly confined system mimics a self-

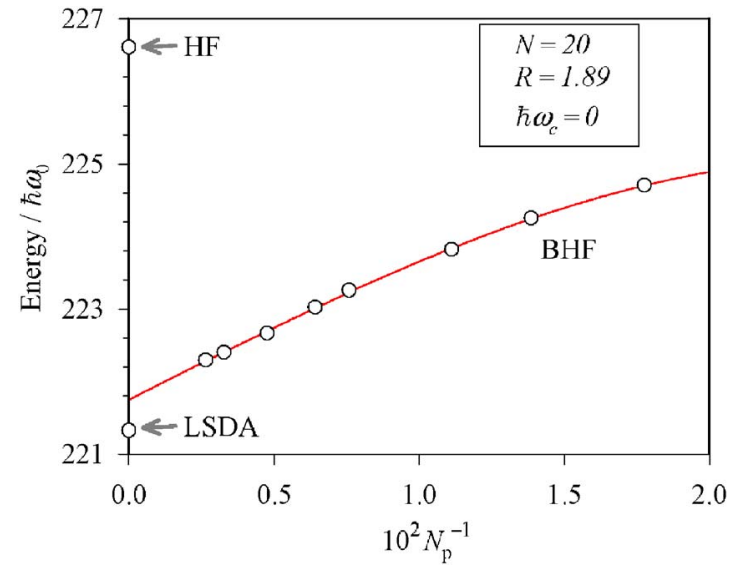

FIG. 5. (Color online) Same as Fig. 4 for a dot with 20 electrons and the additional parameters given in the inset.

assembled InAs dot with $\hbar \omega_{0}=50 \mathrm{meV}$. Following Ref. 32 we take for this material $m^{*}=0.024 m_{e}$ and $\epsilon=15.15$ giving, for $N=4$, a small Wigner-Seitz radius of $r_{s} \sim 0.12$.

To emphasize the possibility of calculating the energies of larger systems in BHF theory we end this section by showing in Fig. 5 the results for an $N=20$ dot. For this number of electrons exact methods like CI or QMC become extremely demanding and we have not attempted to compare with them. The evolution of the BHF energy with the number of particle states resembles that of Fig. 4 although, as one could expect, larger values of $N_{p}$ need to be considered for a similar degree of convergence. The proximity of the extrapolated-BHF and LSDA energies for $N=20$ is a bit surprising since, as shown in Fig. 3, for six electrons in the same confinement the difference is larger. BHF correlations are thus a more important contribution for $N=20$ than for $N=6$. This can be understood as a different degree of magicity for these two dots. Indeed, a highly magic system is characterized by a distribution of single-particle orbitals whose energies group in bunches corresponding to quasi-degenerate shells, with large energy gaps between the shells. One expects a quenching of independent-pair motions in a highly magic system, with respect to collective motions, and, therefore, a relatively worse performance of the BHF theory for them. Note also that since the 20-electron dot has a higher density than the six-electron dot for the same confinement, a better performance of the BHF theory in the former agrees with our preceding results regarding the high density limit.

\section{CONCLUSIONS}

We have explored the prediction of the BHF theory for the correlation energy in two-dimensional parabolic dots. Rather than attempting systematic calculations we intend to point out characteristic trends when applying a well established many body approach such as the BHF theory to parabolic quantum dots. By comparing with exact calculations we have quantitatively discussed the relevance of the BHF correlations as a function of the confinement potential and applied magnetic field for several quantum dots. The BHF theory converges to the exact correlation energy in the limit 
of strong confinement potential (small $R$ parameter, high densities). Also relevant is the limit of strong magnetic fields where the MDD is formed. In these two regions the standard LSDA is shown to be grossly inadequate while BHF stands as a competitive method that sizeably improves on the HF theory. In general, the BHF correlation energy gathers contributions from many empty HF states, as evidenced by the smooth convergence with the space dimension.

Possible extensions of the calculations presented here can consider (i) relaxing the circular symmetry constraint, and (ii) including self-consistency in the BHF single particle orbitals. We shall address the latter by finding the improved mean field proposed by Bethe, Brandow, and Petschek ${ }^{39}$ that actually implies the solution of a double self-consistency problem, on orbitals and effective interaction. Work along these lines is now in progress.

\section{ACKNOWLEDGMENTS}

This work was supported by Projects No. PRIB-20049765 and FIS2005-02796 (L1.S.). A.E. acknowledges support from the Generalitat de Catalunya. E.L. has been supported by DGU (Spain) Grant No. SAB2004-0091.
*Permanent address: Dipartimento di Fisica, Università di Trento, and INFN sezione di Trento, I-38050 Povo, Italy.

${ }^{1}$ U. Meirav, M. A. Kastner, and S. J. Wind, Phys. Rev. Lett. 65, 771 (1990).

${ }^{2}$ R. C. Ashoori, H. L. Stormer, J. S. Weiner, L. N. Pfeiffer, S. J. Pearton, K. W. Baldwin, and K. W. West, Phys. Rev. Lett. 68, 3088 (1992).

${ }^{3}$ S. Tarucha, D. G. Austing, T. Honda, R. J. van der Hage, and L. P. Kouwenhoven, Phys. Rev. Lett. 77, 3613 (1996); Jpn. J. Appl. Phys., Part 1 36, 3917 (1997); S. Sasaki, D. G. Austing, and S. Tarucha, Physica B 256, 157 (1998).

${ }^{4}$ D. G. Austing, S. Sasaki, S. Tarucha, S. M. Reimann, M. Koskinen, and M. Manninen, Phys. Rev. B 60, 11514 (1999).

${ }^{5}$ A. Kumar, S. E. Laux, and F. Stern, Phys. Rev. B 42, 5166 (1990).

${ }^{6}$ M. Fujito, A. Natori, and H. Yasunaga, Phys. Rev. B 53, 9952 (1996).

${ }^{7}$ H. M. Muller and S. E. Koonin, Phys. Rev. B 54, 14532 (1996).

${ }^{8}$ C. Yannouleas and U. Landman, Phys. Rev. Lett. 82, 5325 (1999).

${ }^{9}$ B. Reusch and H. Grabert, Phys. Rev. B 68, 045309 (2003).

${ }^{10}$ L1. Serra, R. G. Nazmitdinov, and A. Puente, Phys. Rev. B 68, 035341 (2003); A. Puente, Ll. Serra, R. G. Nazmitdinov, ibid. 69, 125315 (2004).

${ }^{11}$ M. Eto, Jpn. J. Appl. Phys., Part 1 36, 3924 (1997).

${ }^{12}$ P. A. Maksym and T. Chakraborty, Phys. Rev. Lett. 65, 108 (1990).

${ }^{13}$ D. Pfannkuche, V. Gudmundsson, and P. A. Maksym, Phys. Rev. B 47, 2244 (1993).

${ }^{14}$ P. Hawrylak and D. Pfannkuche, Phys. Rev. Lett. 70, 485 (1993).

${ }^{15}$ J. J. Palacios, L. Martin-Moreno, G. Chiappe, E. Louis, and C. Tejedor, Phys. Rev. B 50, 5760 (1994).

${ }^{16}$ T. Ezaki, N. Mori, and C. Hamaguchi, Phys. Rev. B 56, 6428 (1997).

${ }^{17}$ A. Emperador, E. Lipparini, and F. Pederiva, Phys. Rev. B 72, 033306 (2005).

${ }^{18}$ A. Harju, V. A. Sverdlov, R. M. Nieminen, and V. Halonen, Phys. Rev. B 59, 5622 (1999).

${ }^{19}$ J. Shumway, L. R. C. Fonseca, J. P. Leburton, R. M. Martin, and D. M. Ceperley, Physica E 8, 260 (2000).

${ }^{20}$ F. Bolton, Phys. Rev. B 54, 4780 (1996).

${ }^{21}$ C. H. Mak, R. Egger, and H. Weber-Gottschick, Phys. Rev. Lett. 81, 4533 (1998); R. Egger, W. Hausler, C. H. Mak, and H.
Grabert, ibid. 82, 3320 (1999).

${ }^{22}$ F. Pederiva, C. J. Umrigar, and E. Lipparini, Phys. Rev. B 62, 8120 (2000).

${ }^{23}$ C. Yannouleas and U. Landman, J. Phys.: Condens. Matter 14, L591 (2002); C. Yannouleas and U. Landman, Phys. Rev. B 69, 113306 (2004).

${ }^{24}$ M. Koskinen, M. Manninen, and S. M. Reimann, Phys. Rev. Lett. 79, 1389 (1997).

${ }^{25}$ K. Hirose and N. S. Wingreen, Phys. Rev. B 59, 4604 (1999).

${ }^{26}$ M. Ferconi and G. Vignale, Phys. Rev. B 50, 14722 (1994); E. Lipparini, N. Barberan, M. Barranco, M. Pi, and Ll. Serra, Phys. Rev. B 56, 12375 (1997); M. Pi, M. Barranco, A. Emperador, E. Lipparini, and Ll. Serra, ibid. 57, 14783 (1998); O. Steffens, U. Rossler, and M. Suhrke, Europhys. Lett. 42, 529 (1998); 44, 222 (1998).

${ }^{27}$ B. T. Miller, W. Hansen, S. Manus, R. J. Luyken, A. Lorke, J. P. Kotthaus, S. Huant, G. Medeiros-Ribeiro, and P. M. Petroff, Phys. Rev. B 56, 6764 (1997).

${ }^{28}$ R. J. Warburton, B. T. Miller, C. S. Dürr, C. Bödefeld, K. Karrai, J. P. Kotthaus, G. Medeiros-Ribeiro, P. M. Petroff, and S. Huant, Phys. Rev. B 58, 16221 (1998).

${ }^{29}$ D. Bimberg, M. Grundmann, and N. N. Ledentsov, Quantum dot heterostructures (Wiley, England, 1999), Chap. 8.

${ }^{30}$ B. Szafran, J. Adamowski, and S. Bednarek, Phys. Rev. B 61, 1971 (2000).

${ }^{31}$ S.-J. Cheng, W. Sheng, and P. Hawrylak, Phys. Rev. B 68, 235330 (2003).

${ }^{32}$ T. Brocke, M. T. Bootsmann, M. Tews, B. Wunsch, D. Pfannkuche, Ch. Heyn, W. Hansen, D. Heitmann, and C. Schüller, Phys. Rev. Lett. 91, 257401 (2003).

${ }^{33}$ M. A. Preston and R. K. Badhuri, Structure of the nucleus (Addison-Wesley, Reading, Massachusetts, 1982), Chap. 8.

${ }^{34}$ A. L. Fetter and J. D. Walecka, Quantum theory of many-particle systems (McGraw-Hill, New York, 1971).

${ }^{35}$ K. A. Brueckner, J. L. Gammel, and H. Weitzner, Phys. Rev. 110, 431 (1958); J. W. Negele, Phys. Rev. C 1, 1260 (1970).

${ }^{36}$ E. Lipparini, Ll. Serra, and K. Takayanagi, Phys. Rev. B 49, 16733 (1994).

${ }^{37}$ R. Pérez and A. González, Physica E (Amsterdam) 8, 91 (2000).

${ }^{38}$ B. Tanatar and D. M. Ceperley, Phys. Rev. B 39, 5005 (1989).

${ }^{39}$ H. A. Bethe, B. H. Brandow, and A. G. Petschek, Phys. Rev. 129, 225 (1963). 